\title{
Fatty acid composition, fat deposition, lipogenic gene expression and performance of broiler fed diet supplemented with different sources of oil
}

\begin{abstract}
The present study assessed the effect of feeding palm oil (PO), sunflower oil (SO) and their combination on performance, fat deposition, fatty acid composition and lipogenic gene expression of broilers reared for 42 days. A total of 144 1-day-old broilers (Cobb500) were randomly allotted into four treatment diets with each having six replicates of six chicks in each replicate following a completely randomized design. Live weight gain and feed efficiency was significantly $(\mathrm{P}<0.05)$ higher in birds fed with a combination of oil sources compared to controls. Birds fed with the combination of oil and $\mathrm{SO}$ alone had higher carcass yield and lower abdominal fat. Higher $(\mathrm{P}<0.05)$ concentrations of unsaturated fatty acids (UFA) and lower concentrations of palmitic acid and saturated fatty acid (SFA) was found in birds fed SO alone and combinations of SO and PO. Furthermore, the outcomes showed that birds fed diet supplemented with SO and the combination of SO and PO down-regulated gene expression of key hepatic lipogenic enzymes of fatty acids synthase (FAS), acetyl-CoA carboxylase (ACC) and stearoyl-CoA desaturase (SCD). These findings suggest that the diet containing the combination of $2 \% \mathrm{PO}$ and $4 \%$ SO may reduce hepatic lipogenesis, as well as lower abdominal fat content of broilers.
\end{abstract}

Keyword: Dietary fat; Fatty acid synthase; Gene expression; Lipogenesis 